\title{
Analytic studies on fetal heart rate changes to prevent cerebral palsy with novel hypoxia index in fetal monitoring
}

\author{
Kazuo Maeda* \\ Honorary Professor, OBGY, Tottori University medical school, Yonago, Japan
}

\begin{abstract}
Fetal heart rate (FHR) rises when the fetus moves, where FHR acceleration is reacted fetal brain to fetal movements (burst), it losts in early fetal hypoxia, while FHR variability is the reaction to minor fetal motions, which losts in severe hypoxia followed by cerebral palsy.

FHR falls in hypoxia when fetal $\mathrm{PaO}_{2}$ is 50 or less $\mathrm{mmHg}$, while fetal $\mathrm{PaO}_{2}$ is lower than 50 mmHg, where fetal vagal nerve center is excited by hypoxia, forming FHR bradycardia and deceleration. The late deceleration was ominous in the past, while 3 connective typical late decelerations' outcome was normal, while repeated late deceleraions for 50 minutes developed heavy asphyxia and severe brain damage, thus, novel hypoxia index is the sum of deceleration durations (min) divided by the lowest FHR, and multiplied by 100, where no cerebral palsy developed when hypoxia index was 24 or less, while the index was 25 or more in cases of cerebral palsy. Visual FHR patttern classification will be changed to objective hypoxia index and FHR score which predicts Apgar score and UApH. FHR frequency spectrum diagnoses pathologic sinusoidal FHR.caused by severe fetal anemia imminent to fetal death.
\end{abstract}

\section{Introduction}

The fetal heart rate (FHR) is the target of intrapartum fetal monitoring, where main subject was abnormal FHR pattern, including bradycaedia, the loss of baseline variability followed by cerebral palsy, late and severe variable decelerations and pathologic sinusoidal FHR, mainly diagnosed by visual classification during delivery. Abnormal FHR was treated by oxygen inhalation, posture change of parturient woman to lateral one, and main therapy was caesarean early delivery. Perinatal mortality and cerebral palsy were reduced in Japan while Dublin trial did not show the reduction of cerebral palsy, thus, update target is the reduction of cerebral palsy.

\section{Methods}

Although main target was the detection of FHR pattern abnormality to perform emergency early caesarean delivery, particularly late and severe variable decelerations. Some abnormal FHR classification placed the loss of baseline variability at the worst FHR change, however, the loss of variability resulted unexpected cerebral palsy, namely, the loss of variability was the mostly severe hypoxic fetal brain damage. Although late deceleration was severely abnormal pattern in abnormal FHR pattern [1], the author experienced fully normal, Apgar 9 neonate was born by caesarean delivery, after 3 connected typical late decelerations in the past, while frequently repeated late deelerations in 50 minutes due to refusal of caesarean delivery, the results was severly asphyxiated Apgar 3 neonate with severe infantile brain damage. In addition, a late deceleration definition needed 15 or more minutes' repetition of the decelerations before definition of late deceleration, thus the author understood that late decelerations is ominous not due to the late appearance of deceleration, but frequent repetition of FHR decelerations may develop severe fetal hypoxia, namely, the hypoxia stimulate and excited vagal nerve center located in medulla oblongata, developing FHR bradycardia, where the heart rate was lineally parallel to $\mathrm{PaO}_{2}$, if the $\mathrm{PaO}_{2}$ is lower than $50 \mathrm{mmHg}$ [2] and actuall human fetal $\mathrm{PaO}_{2}$ was 50 or less mmHg [3]. Thus, small number of FHR deceleration will not affect the fetus, while frequently repeated decelerations will damage the fetus. The author intended to observe the damage by the novel hypoxia index as follows; Novel hypoxia index=the sum of all deceleration durations ( $\mathrm{min}$ ) in fetal monitoring, divided by the lowest FHR (bpm), and multiplied by 100 to keep the index an integer. The authors collected 6 cases of infatile cerebral palsy, and 16 cases of no cerebral palsy diagnosed in pediatric clinics and calculated their hypoxia indexes in FHR recorded in intrapartum monitoring preserved in Obstetric ward.

\section{Results and discussion}

The hyoxia index of cerebral palsy was 25 or more in all of 6 cerebral palsy cases, while the index was 24 or less in all of 16 cases of no cerebral palsy, and there was significant difference. As the probability to make wrong decision was 0.000008 , almost zero in the $\mathrm{Chi}^{2}$ test, we decided the case whose hypoxia index is 24 or less predicted no cerebral palsy, namely, infantile cerebral palsy is prevented when the novel hypoxia index is 24 or less.

Also, the case, whose hypoxia index was 25 or more, can receive the cerebral palsy treatment early, even in neonatal stage.

Furthermore, increasing hypoxia index in fetal monitoring can be prevented by the change to Lateral posture posture from supine

${ }^{\star}$ Correspondence to: Kazuo Maeda, MD, PhD, 3-125 Nadamachi, Yonago, Tottoriken, 683-0835, Japan, Tel: +81859226856; E-mail: maedak@mocha.ocn.ne.jp

Key words: fetal heart rate, hypoxia index, fhr score, fhr frequency spectrum, fhr pattern classification, late deceleration

Received: February 04, 2019; Accepted: February 15, 2019; Published: February 19,2019 
posture, when FHR deceleration is recorded on the FHR curve in fetal monitoring, because it was reported that FHR deceleration disappeared when the parturient woman changed supine posture to lateral one [4], namely, hypoxia index does not increase if the deceleration is lost.

Although novel hypoxia index is able to be manually calculated, automatic calculation with computer will be more convenient in the future, where already Apgar score and UApH are predicted by the FHR score [5], and pathologic sinusoidal FHR which appears in severe fetal anemia and asphyxia imminent to fetal demise will also be predicted by computerized FHR curve frequency spectrum, thus, visual FHR chart diagnosis with FHR pattern classification will be replaced by computerized mathematical FHR diagnosis in the future.

We have to recognize that intrauterine fetal environment is hypoxic, namely fetal blood $\mathrm{PaO}_{2}$ is lower than $50 \mathrm{mmHg}$, which is lower than half of adult arterial blood $\mathrm{PaO}_{2}$ which will be 100 $\mathrm{mmHg}$ or more. Thus, a fetus is easily damaged by hypoxia. We have to recognize that FHR deceleration is the hypoxic sign lower than $50 \mathrm{mmHg}$. Unfortuntely the relation of human fetal $\mathrm{PaO}_{2}$ and fetal heart rate is unknown. It is an estimation based on rabbit experiment, namely, feftal deceleration hypoxia is estimated from rabbit's case [2].

\section{Disclosure}

None

\section{Conclusion}

It is lucky to know that there is no cerebral palsy if hypoxia index is 24 or less. We can prevent cerebral palsy keeping hypoxia index at 24 or less level. Lateral posture at appearance of deceleration will promote to keep hypoxia index at low level.

As Happier world will appear when handicapped children decrease by advanced fetal monitoring reducing cerebral palsy by novel hypoxia index. The index and similar mathematical fetal diagnosis will change conventional FHR pattern classification, where update FHR diagnosis is clearly preventing cerebral palsy to improve young happy lives in the world.

\section{References}

1. Hon EH (1998) An atlas of fetal heart rate patterns. Narty Press, New Haven.

2. Umezawa $\mathbf{J}$ (1975) Studies on the relation between heart rate and $\mathrm{PaO}_{2}$ in hypoxic rabbit: a comparative study

3. for fetal heart rate change in labor. Acta Obstet Gynecol Jpn 28: 1203-1212.

4. Maeda K, Kimura S, Nakano H (1969) Pthophysiology of Fetus, Fukuoka Printing, Fukuoka.

5. Poseiro JJ, Mendez-Bauer C, Caldeyro-Barcia R (1969) Effect of uterine contractions on maternal blood flow through the placenta. Perinatal factors affecting human development. Paho Advisary Committee 161-171.

6. Maeda K, Noguchi Y, Matsumoto F, Nagasawa T (2006) Quantitative fetal heart rate evaluation without pattern classification: FHR score and artificial neural network analysis. In Kurjak, Chervenak Eds. Textboook of Perinatal Medicine, $2^{\text {nd }}$ ed. London, Informa Network 21: 127-141 [Crossref]

Copyright: (C2019 Maeda K. This is an open-access article distributed under the terms of the Creative Commons Attribution License, which permits unrestricted use, distribution, and reproduction in any medium, provided the original author and source are credited. 